\title{
ON THERMAL INSTABILITY IN RIGID HEAT CONDUCTORS WITH NONLINEAR HEAT GENERATION*
}

\author{
By PETER J. CHEN (Sandia Laboratories, Albequerque) \\ R. RAY NACHLINGER (University of Houston) \\ IND JACE W. NUNZIATO (Sandia Laboratories, Albequerque)
}

1. Introduction. In this paper we consider the stability properties of the temperature $\theta$ in rigid heat conductors with positive, nonlinear heat generation $g$, without proposing any explicit representation for $g$. We restrict our attention to the initial-value problem for finite domains and derive time-dependent upper and lower bounds for the solution. These bounds depend on the initial temperature in the body and on the properties of the heat generation in the range of the solution. In particular, we show that if $g$ is bounded from above by a linear function whose slope is equal to the initial slope of $g$, then the solution always seems to be bounded from above. However, this is not the case if $g$ is bounded from below by the same linear function. In this situation, the temperature may grow without bound if either (i) the initial slope of $g$ exceeds a number $\lambda$ which is the smallest positive eigenvalue of the homogeneous eigenvalue problem associated with the domain of the body, or (ii) the maximum initial temperature exceeds a positive number $\eta_{m}$ which we call the critical temperature. This possible unbounded growth of the temperature is interpreted as a thermal instability. Such instabilities have been observed in chemically reacting solids and are often referred to as thermal explosions.

Our study is motivated by the properties of the solution when the heat generation is taken to be that which arises for the case of chemically reacting media with Arrhenius kinetics. In this situation, the heat generation is proportional to $e^{-1 / T}$, where $T$ is some non-dimensional temperature. Notice that $e^{-1 / T}$ is concave from above whenever $T<1 / 2$ and it is convex from above whenever $T>1 / 2$. Several authors have been able to establish the existence of the critical temperature only when the maximal solution is less than 1/2 everywhere. (See, for example, Enig [1], Enig, Shanks and Southworth [2], and Bailey [3].) This leads one to suspect that the stability properties of the solution must depend on the properties of the heat generation.

2. Bounds on the temperature field. Let $D$ denote the interior of a bounded regular region in Euclidean 3-space, and let $\partial D$ denote its boundary. We identify the material body with $\bar{D}=D \cup \partial D$. Further, let $\mathbb{R}$ denote the interval $\left[0, t_{0}\right.$ ) for some $t_{0}>0$ (possibly $\left.t_{0}=+\infty\right)$ and $R$ the interval $\left(0, t_{0}\right)$. An element of $\bar{D} \times \mathfrak{R}$ is denoted by the pair $(\mathbf{x}, t)$.

* Received March 8, 1975. The authors wish to thank R. N. Rogers and W. N. Sullivan for stimulating discussions during the early stages of this study. Thanks are also due to P. B. Bailey for helpful discussions regarding certain aspects of our work. This work was supported by the U.S. Atomic Energy Commission and the U.S. National Science Foundation. 
The temperature field in a rigid heat conductor with nonlinear heat generation is governed by the equation

$$
\dot{\theta}=\nabla^{2} \theta+g(\theta),
$$

where $\theta$ is the temperature difference $\varphi-\varphi_{0}$ with $\varphi$ being the absolute temperature and $\varphi_{0}$ being some reference temperature. We assume that the heat generation g has the following properties:

$$
g(0)=0, \quad g(\theta)>0, \quad 0<g^{\prime}(\theta) \leq M,
$$

where $M$ is some finite positive number. Let $f$ be a function defined on $\bar{D}$ such that $f$ is of class $C^{2}$ on $D, f(\mathbf{x})>0$ for every $\mathbf{x} \in D$, and $f(\mathbf{x})=0$ for every $\mathbf{x} \in \partial D$. The function $\theta$ defined on $\bar{D} \times \Re$ and of class $C^{2}$ on $D \times R$ is said to be a solution of the initialvalue problem if it satisfies the differential equation (2.1) with

$$
\begin{array}{lll}
\theta=0 & \text { on } & \partial D \times R, \\
\theta=f & \text { on } & D \times\{0\} .
\end{array}
$$

The existence of a unique solution for this problem is well established. (See, for example, Walter [4], §25.) Of course, if $f \equiv 0$ on $D$, then $\theta \equiv 0$ on $\bar{D} \times \mathfrak{R}$ is a solution of (2.1)(2.3); we call this the zero solution.

In the sequel we shall examine the stability properties of the zero solution associated with the initial-value problem (2.1)-(2.3). $\dagger$ It will become evident that these properties are strongly dependent on the properties of the heat generation $g$ in the range of the solutions. The following lemma is essential to the proofs of our main results. (Cf. Walter $[4], \S 24$.)

Lemma 1. Let $\psi$ and $\rho$ be functions defined on $D \times \Re$ such that $\psi$ and $\rho$ are of class $C^{2}$ on $D \times R$, and let

$$
\begin{gathered}
P \psi \equiv \dot{\psi}-\nabla^{2} \psi-g(\psi), \\
P \rho \equiv \dot{\rho}-\nabla^{2} \rho-g(\rho) .
\end{gathered}
$$

If $g$ satisfies $\left(2.2_{3}\right)$ and if

(i) $\rho \leq \psi$ on $\partial D \times R$ and $D \times\{0\}$,

(ii) $P \rho \leq P \psi$ on $D \times R$, then

$$
\rho \leq \psi \quad \text { on } \quad \bar{D} \times \mathfrak{R} .
$$

We are now in the position to prove our main results. Our first result deals with the case when the heat generation $g$ is finite and bounded below by a linear function in the range of the solution $\theta$.

Theorem 1. Let $\theta$ be a solution of the initial-value problem. Suppose that there exists a finite positive number $\beta^{+}$such that $2 \beta^{+} \geq \max g^{\prime \prime}(\theta)$ and that in the range of the solution

$$
\alpha \theta \leq g(\theta) \leq \alpha \theta+\beta^{+} \theta^{2},
$$

+ The stability of the temperature when $g(\theta)<0$ has been discussed by Bellman [5], Narasimhan [6], and Greenberg [7]. The differential equation (2.1) also governs the stability of reaction-diffusion systems and has been studied by Georgakis and Sani $[8]$ for the situation when $g$ is represented by a fourth-order polynomial in $\theta$. 
where $\alpha=g^{\prime}(0)$. Further, suppose that

(i) there exist positive numbers $\delta, \lambda, \gamma^{-}, \gamma^{+}$, and functions $U, V$ defined on $\bar{D}$ and of class $C^{2}$ on $D$ such that

$$
\begin{array}{lll}
\nabla^{2} U \leq-\delta U, & \gamma^{-} \leq U^{\top} \leq \gamma^{+} \quad \text { on } \quad \bar{D}, \\
\nabla^{2} V=-\lambda V, & 0<V \text { on } D, \quad V=0 \quad \text { on } \partial D,
\end{array}
$$

and

(ii) there exist functions $\mathrm{H}, G$ defined on $\mathfrak{a}$ and of class $C^{1}$ on $R$ such that

$$
\begin{aligned}
\dot{H} & =(\alpha-\delta) H+\beta^{+} \gamma^{+} H^{2}, \\
\dot{G} & =(\alpha-\lambda) G .
\end{aligned}
$$

Then the solution $\theta$ is bounded according to

$$
V\left(i(0) \exp (-\xi t) \leq \theta \leq \frac{\mu / \beta^{+}}{\left\{\frac{\eta_{1}}{\max f}-1\right\} \exp (\mu t)+1} \text { on } \bar{D} \times \mathfrak{R},\right.
$$

where $V G(0) \leq f$ on $D$, and

$$
\xi=\lambda-\alpha, \quad \mu=\delta-\alpha, \quad \eta_{1}=\gamma^{-} \mu / \beta^{+} \gamma^{+} .
$$

Proof. We shall first establish the upper bound of (2.7). Clearly $\left(2.6_{1}\right)$ together with the initial condition

$$
H(0)=\frac{1}{\gamma^{-}} \max f
$$

implies that $H$ is positive-valued. By $\left(2.6_{1}\right)$,

$$
U \dot{H}=(\alpha-\delta) U H+\beta^{+} \gamma^{+} U H^{2} .
$$

But, in view of $\left(2.5_{1}\right)$,

$$
U \leq \gamma^{+}, \quad \nabla^{2} U \leq-\delta U
$$

Hence (2.10) implies that

$$
U \dot{H} \geq \nabla^{2}(U H)+\alpha U H+\beta^{+}(U H)^{2} .
$$

Letting $\psi=U H$, we have

$$
\dot{\psi} \geq \nabla^{2} \psi+\alpha \psi+\beta^{+} \psi^{2} .
$$

Since $\psi$ is positive-valued, it follows from (2.4) and (2.11) that

$$
\begin{aligned}
P \psi & =\dot{\psi}-\nabla^{2} \psi-g(\psi) \\
& \geq \alpha \psi+\beta^{+} \psi^{2}-g(\psi) \\
& \geq 0 .
\end{aligned}
$$

Thus, since $\theta$ is a solution of the initial-value problem, $P \theta=0$; consequently,

$$
P \theta \leq P \psi \text { on } D \times R .
$$


Also, (2.3), (2.5 $\left.5_{1}\right),\left(2.6_{1}\right)$ and (2.9) imply that

$$
\theta \leq \psi \quad \text { on } \quad \partial D \times R \text { and } D \times\{0\} .
$$

Thus the hypotheses of Lemma 1 are satisfied and we conclude that

$$
\theta \leq \psi \quad \text { on } \bar{D} \times \mathfrak{R} .
$$

The proof of the upper bound is completed by integrating the differential equation $\left(2.6_{1}\right)$ for $H$ and recalling that $\psi=U H \leq \gamma^{+} H$.

The proof of the lower bound follows an entirely analogous argument; that is, we simply let $\rho=V G$ with $V G(0) \leq f$ on $D$, and show that $\rho \leq \theta$ on $\bar{D} \times \Re$.

Our subsequent results deal with the case when the heat generation $g$ is bounded above by a linear function in the range of the solution.

Theorem 2. Let $\theta$ be a solution of the initial-value problem. Suppose that there exists a finite positive number $\beta^{-}$such that $2 \beta^{-} \leq \min \left|g^{\prime \prime}(\theta)\right|$ and that in the range of the solution

$$
g(\theta) \leq \alpha \theta-\beta^{-} \theta^{2},
$$

where $\alpha=g^{\prime}(0)$. Further, suppose that

(i) there exist positive numbers $\delta, \gamma^{-}, \gamma^{+}$, and a function $U$ defined on $\bar{D}$ and of class $C^{2}$ on $D$ such that

$$
\nabla^{2} U \leq-\delta U, \quad \gamma^{-} \leq U \leq \gamma^{+}
$$

and

(ii) there exists a function $F$ defined on $\Re$ and of class $C^{1}$ on $R$ such that

$$
\dot{F^{\prime}}=(\alpha-\delta) F-\beta^{-} \gamma^{-} F^{2} .
$$

Then the solution $\theta$ is bounded from above according to

$$
\theta \leq \frac{\eta_{2} \gamma^{+} / \gamma^{-}}{\left\{-\frac{\eta_{2}}{\max f}+1\right\} \exp (\mu t)-1} \text { on } \bar{D} \times \mathfrak{R},
$$

where

$$
\mu=\delta-\alpha, \quad \eta_{2}=\mu / \beta^{-} .
$$

Proof: The proof of (2.12) is entirely analogous to the proof of the upper bound of Theorem 1; i.e., we simply let $\psi=U F$ with $\gamma^{-} F(0)=\max f$ and show that $\theta \leq \psi$ on $\bar{D} \times \Re$.

Remark 1. If $\beta^{-}=0$, it is a simple matter to show that $(2.12)$ reduces to

$$
\theta \leq{\frac{\gamma^{+}}{\gamma}}^{+}(\max f) \exp (-\mu t)
$$

with $\mu=\delta-\alpha$. This same result follows from (2.7) when $\beta^{+}=0$. It is obvious that (2.14) holds for the case when $g(\theta) \leq \alpha \theta$. (This case was discussed previously by Walter [4], §28. XII.)

Our last result concerns the situation when

$$
\alpha \theta-\beta_{+} \theta^{2} \leq g(\theta) \leq \alpha \theta
$$


with $2 \beta_{+} \geq \max \left|g^{\prime \prime}(\theta)\right|$. In view of the preceeding remark we shall only prove the following:

Theorem 3. Let $\theta$ be a solution of the initial-value problem. Suppose that there exists a finite positive number $\beta_{+}$such that $2 \beta_{+} \geq \max \left|g^{\prime \prime}(\theta)\right|$ and that in the range of the solution

$$
\alpha \theta-\beta_{+} \theta^{2} \leq g(\theta)
$$

where $\alpha=g^{\prime}(0)$. Further, suppose that

(i) there exist positive numbers $\lambda, \omega$, and a function $V$ defined on $\bar{D}$ and of class $C^{2}$ on $D$ such that

$$
\nabla^{2} V=-\lambda V, \quad 0<V \leq \omega \text { on } D, \quad V=0 \text { on } \partial D
$$

and

(ii) there exists a function I defined on $\mathbb{R}$ and of class $C^{1}$ on $R$ such that

$$
\dot{I}=(\alpha-\lambda) I-\beta_{+} \omega I^{2} .
$$

Then the solution is bounded from below according to

$$
\frac{V \eta_{3} / \omega}{\left\{\frac{\eta_{3}}{\omega I(0)}+1\right\} \exp \xi t-1} \leq \theta \text { on } \bar{D} \times \mathfrak{k}
$$

where

$$
\xi=\lambda-\alpha, \quad \eta_{3}=\xi / \beta_{+} .
$$

Proof. To prove (2.15) we simply let $\rho=\mathrm{VI}$ with $\mathrm{VI}(0) \leq f$ on $D$ and show that $\rho \leq \theta$ on $\bar{D} \times \mathfrak{R}$.

Remark 2. If $\beta_{+}=0,(2.15)$ reduces to the lower bound given by (2.7), i.e., for the case when $\alpha \theta \leq g(\theta)$.

3. Discussion of stability behavior. Here we examine the implications of the theorems given in the preceding section with regard to the stability properties of the zero solution. Let us first consider the results of Theorem 1 for which the heat generation $g$ is bounded below by a linear function in the range of the solution.

Consider the eigenvalue problem

$$
\nabla^{2} V+\lambda V=0 \text { on } D, \quad V=0 \text { on } \partial D .
$$

and let $\lambda$ be the smallest eigenvalue for which a positive solution of (3.1) exists. Since the eigenvalues depend continuously on the region under consideration, there exists an arbitrary region $D^{*} \supset D$ such that the smallest eigenvalue $\delta$ corresponding to the eigenvalue problem

$$
\nabla^{2} U+\delta U=0 \text { on } D^{*}, \quad U=0 \text { on } \partial D,
$$

is less than $\lambda$. If the initial slope $\alpha$ of the heat generation is greater than the eigenvalue $\lambda$, so that $\delta<\lambda<\alpha$, then it follows from (2.7) of Theorem 1 that the solution $\theta$ is bounded from above by a function which becomes infinite in a finite time $t^{\infty}$,

$$
t^{\infty}=-\frac{1}{\mu} \ln \left\{1-\frac{\eta_{1}}{\max f}\right\},
$$


and it is bounded from below by a function which becomes infinite as $t \rightarrow \infty$. (It is clear that in this situation the functions $H$ and $G$ in Theorem 1 are defined over the intervals $\left[0, t_{0}\right), t_{0} \leq t^{\infty}$, and $[0, \infty)$, respectively.) This situation does not necessarily imply an unbounded temperature field but it does imply that when $\lambda\langle\alpha$ and $g(\theta)>\alpha \theta$ in the range of the solution there is temperature growth. We interpret this behavior as a thermal instability.

Consider now the case when the initial slope $\alpha$ of $g$ is less than the eigenvalue $\lambda$. In this instance, the lower bound of the solution $\theta$ tends to zero as $t \rightarrow \infty$. However, the behavior of the upper bound is more complicated and depends on the choice of the region $D^{*}$. We first note that there are infinitely many choices of the region $D^{*}$ such that the conditions (2..5) of Theorem 1 is satisfied. We further note that since the bounds $\gamma^{-}$ and $\gamma^{+}$of $l$ depend on the choice of $D^{*}$ they can be viewed as continuous functions of $\delta$. Consequently, for a given heat generation $g, \eta_{1}$, defined by $\left(2.8_{3}\right)$, is given by

$$
\eta_{1}=\eta_{1}(\delta) \text {. }
$$

Thus, if wr can find a region $D^{*}$ such that $\alpha<\delta<\lambda$ and

$$
\eta_{1}>\max f
$$

then, by (2.7), the upper bound also tends to zero as $t \rightarrow \infty$. In this case, the zero solution is said to be asymptotically stable. On the other hand, if we can find a region $D^{*}$ such that $\alpha<\delta<\lambda$ and

$$
\eta_{1}=\max f,
$$

and none for which $\eta_{1}>\max f$, then, by (2.7), we see that the upper bound of the solution is a constant, $\mu / \beta^{+}$. In this case, the zero solution is said to be stable. Finally, if for every choice of the region $D^{*}$ such that $\alpha<\delta<\lambda$ we find that

$$
\eta_{1}<\max f
$$

then, by (2.7), it is clear that the upper bound becomes infinite in a finite time given by (3.3). We cannot say for certain that the zero solution is unstable in this case because the lower bound tends to zero; however, we conjecture that it is on the basis of physical reasoning.

The preceding results suggest that we call the number $\eta_{m}$, defined by

$$
\eta_{m}=\max \eta_{1}(\delta) \text {, }
$$

the eritical temperature; for when $\alpha<\delta<\lambda$, the stability of the zero solution seems to depend on whether the maximum initial temperature is greater than, equal to, or less than $\eta_{m} .^{*}$

We now turn to the examination of the situation when the heat generation $g$ is bounded above by a linear function in the range of the solution.

First, let us consider the implications of the results of Theorem 2. By (2.12), it is a simple matter to show that when $\delta<\lambda<\alpha$ the solution is always bounded by the con-

* For explosives, the existence of a critical temperature has been observed experimentally in that when the temperature exceeds the critical value, a thermal explosion results. We believe that our results may serve to determine this critical temperature. Also, we should point out that since we always seek the minimal upper bound and since the region $D^{*}$ is arbitrary, the case $\delta<\alpha<\lambda$ is naturally excluded from our considerations. 
stant $-\eta_{2} \gamma^{+} / \gamma^{-}$, and we say that the zero solution is stable. On the other hand, if $\alpha<\delta$ $<\lambda$. then the solution is bounded by a function which tends to zero as $t \rightarrow \infty$. That is, the zero solution is asymptotically stable.

Now let us examine the results of Remark 1 and Theorem 3. By (2.14) and (2.15), we see that when $\delta<\lambda<\alpha$ the upper bound of the bounded tends to infinity as $t \rightarrow \infty$, but it always lies above $-V \eta_{3} / \omega$. It is clear that we cannot say anything about the stability properties of the zero solution in this situation even though we suspect that it is stable. Finally, when $\alpha<\delta<\lambda$ both the upper bound and lower bound tend to zero as $t \rightarrow \infty$. Herc, of course, the zero solution is asymptotically stable.

\section{REFERENCES}

[1] J. W. Enig, Approximate solutions in the theory of thermal explosions for semi-infinite explosives, Proc. Roy. Soc. London A 305, 205-217 (1968)

[2] J. W. Enig, D. Shanks, and R. W. Southworth, The numerical solution of the heat conduction cquation occurring in the theory of thermal explosions, U. S. Naval Ordnance Laboratory, NAVORI) Rept. 4377 (1956)

[3] P. B. Bailey, On the problem of thermal instability of explosive materials, Combustion and Flame 23, 329-336 (1974)

[4] W. Walter, Differential and integral inequalities, Springer, New York-Heidelberg-Berlin, 1970

[5] R. Bellman, On the existence and boundedness of solutions of nonlinear partial differential equations of parabolic type, Trans. Amer. Math. Soc. 64, 21-44 (1948)

[6] R. Narasimhan, On the asymptotic stability of solutions of parabolic differential equations, J. Rational Mech. Anal. 3, 303-313 (1954)

[7] J. M. Greenberg, $A$ note on the stability properties of uniform temperature fields, J. Math. Anal. Appl. $19,247-252$ (1967)

[8] C. Georgakis and R. L. Sani, On the stability of the steady state in systems of coupled diffusion and reaction, Arch. Rational Mech. Anal. 52, 266-296 (1973) 